\section{(6) OPEN ACCESS}

\title{
Increased risk of vascular disease associated with gout: a retrospective, matched cohort study in the UK Clinical Practice Research Datalink
}

\author{
Lorna E Clarson, ${ }^{1}$ Samantha L Hider, ${ }_{1}^{1}$ John Belcher, ${ }^{1}$ Carl Heneghan, ${ }^{2}$ \\ Edward Roddy, ${ }^{1}$ Christian D Mallen ${ }^{1}$
}

Handling editor Tore K Kvien

- Additional material is published online only. To view please visit the journal online (http://dx.doi.org/10.1136/ annrheumdis-2014-205252).

${ }^{1}$ Research Institute for Primary Care \& Health Sciences, Keele University, Keele, Staffordshire, UK

${ }^{2}$ Department of Primary Care Health Sciences, Oxford University, Oxford, UK

Correspondence to Dr Lorna Clarson, Research Institute for Primary Care \& Health Sciences, Keele University, Keele, Staffordshire ST5 5BG, UK;

l.clarson@keele.ac.uk

Received 17 January 2014 Revised 1 August 2014 Accepted 2 August 2014 Published Online First 27 August 2014

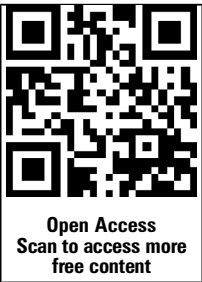

CrossMark

\section{SLinked}

http://dx.doi.org/10.1136 annrheumdis-2014-206432

To cite: Clarson $\mathrm{LE}$, Hider SL, Belcher J, et al. Ann Rheum Dis

2015:74:642-647

\section{ABSTRACT}

Objectives To determine whether gout increases risk of incident coronary heart disease (CHD), cerebrovascular (CVD) and peripheral vascular disease (PVD) in a large cohort of primary care patients with gout, since there have been no such large studies in primary care.

Methods A retrospective cohort study was performed using data from the Clinical Practice Research Datalink (CPRD). Risk of incident CHD, CVD and PVD was compared in 8386 patients with an incident diagnosis of gout, and 39766 age, sex and registered general practicematched controls, all aged over 50 years and with no prior vascular history, in the 10 years following incidence of gout, or matched index date (baseline). Multivariable Cox Regression was used to estimate HRs and covariates included sex and baseline measures of age, Body Mass Index, smoking, alcohol consumption, Charlson comorbidity index, history of hypertension, hyperlipidaemia, chronic kidney disease, statin use and aspirin use.

Results Multivariable analysis showed men were at increased risk of any vascular event (HRs (95\% Cls)) HR 1.06 (1.01 to 1.12), any CHD HR 1.08 (1.01 to 1.15) and PVD HR 1.18 (1.01 to 1.38), while women were at increased risk of any vascular event, HR 1.25 (1.15 to 1.35), any CHD HR 1.25 (1.12 to 1.39), and PVD 1.89 (1.50 to 2.38)) but not any CVD.

Conclusions In this cohort of over 50 s with gout, female patients with gout were at greatest risk of incident vascular events, even after adjustment for vascular risk factors, despite a higher prevalence of both gout and vascular disease in men. Further research is required to establish the reason for this sex difference.

\section{INTRODUCTION}

Gout is the most prevalent inflammatory arthritis, affecting an estimated $2.5 \%$ of the population in the UK, ${ }^{1}$ and $3.9 \%$ in North America. ${ }^{2}$ It is associated with elevated levels of serum uric acid (SUA) and deposition of monosodium urate crystals in tissues and joints, leading to excruciating painful attacks of peripheral joint synovitis which, in the UK, are largely managed in primary care by general practitioners (GP) who refer on for specialised care only if necessary.

Hyperuricaemia, the biochemical precursor to gout, has been linked with an increased incidence of, and mortality from, both CHD and stroke. ${ }^{3} 4$ Although gout is traditionally thought of as an intermittent inflammatory condition, recent ultrasound studies have identified persistent subclinical inflammation in the intercritical period between acute attacks. ${ }^{5}$ It has been hypothesised that the combination of persistent inflammation and hyperuricaemia may potentiate or synergise CHD development. $^{6}$ Deposition of urate crystal material in vessel walls has been proposed to cause neutrophil and platelet activation and release of inflammatory mediators that promote cardiovascular damage. ${ }^{7-9}$

Epidemiological studies examining the relationship between gout and CHD report conflicting findings, with a significant association reported by some, ${ }^{10-13}$ but not others, ${ }^{14-16}$ and investigations of risk of cerebrovascular disease (CVD) or peripheral vascular disease (PVD) in patients with gout comparatively fewer. ${ }^{16-18}$ Consequently, the risk intrinsic to gout itself, compared to that from hyperuricaemia or vascular risk factors, such as hypertension and obesity commonly found in patients with gout, remains unclear. Additionally, many of these studies have been conducted in secondary care populations, who may be characterised by more severe disease, rather than primary care where the majority of patients with gout are managed. ${ }^{19}$

The use of data from routinely collected primary care records is accepted as a cost-effective way to undertake epidemiological studies of large patient populations across a broad population spread. ${ }^{20}$ The UK Clinical Practice Research Datalink (CPRD) is the largest database of electronic primary care health records (EHR) in the world, and has previously been used in the study of the association of inflammatory conditions and vascular disease, ${ }^{21} 22$ as well as in the epidemiology of gout. ${ }^{123}$

We sought to investigate the association between gout and incident CHD, CVD and PVD in a large sample of the UK general practice population.

\section{METHODS}

\section{Clinical practice research datalink}

The UK CPRD contains data for approximately 9\% of the UK population. Currently, 650 general practices contribute high-quality data with over 5.5 million active patients, ${ }^{24}$ thought to be broadly representative of the general UK population, ${ }^{24} 25$ and high validity of diagnosis in the CPRD has been reported. ${ }^{2627}$

\section{Participant identification}

Patients consulting in primary care between 1987 and 1999, with an incident diagnosis of gout, were identified. Potential control subjects with no history 
of gout were stratified by general practice, year of birth and sex, and up to five were randomly selected from the appropriate stratum for each patient with gout. Baseline for patients with gout was considered to be the first entry of a diagnostic code for gout in their EHR, and for patients without gout the date of diagnosis of gout in their matched gout patient. Pre-baseline EHRs were searched for codes relating to prescriptions for colchicine or urate-lowering therapy, and where these were present, the patient record was individually examined to determine whether a prior diagnosis of gout was probable, and subsequent inclusion or exclusion.

Participants in both cohorts were required to be aged over 50 years at baseline, since vascular events themselves are rare under the age of 50 years, and are often influenced by other underlying factors which may be difficult to account for. Patients with a previous history of vascular disease were excluded in order to minimise the surveillance bias associated with follow-up for a previous event, and remove the contribution of additional risk conferred by a previous event.

\section{Outcome measures}

Outcomes of interest were time to first recording of CHD, CVD and PVD within the patient's EHR. Events of interest were angina, myocardial infarction (MI) and any CHD (including angina, MI and all less specific codes describing incident coronary heart disease), stroke/cerebrovascular accident (CVA), transient ischaemic attack (TIA) and any CVD (including TIA, CVA and all less specific codes describing incident cerebrovascular events) and PVD (considered to be any narrowing of arteries distal to the arch of the aorta and identified in the EHR using codes associated with symptoms at incidence of disease, eg, intermittent claudication, or asymptomatic identification by screening in high-risk populations, eg, diabetics). The term 'any vascular event' was used to describe overall risk of developing any of these outcomes of interest. Due to the nature of the coding, we were not able to separate ischaemic from haemorrhagic cerebrovascular events, and for this reason they were considered together. A list of Read Codes used is available on request. Participants were followed from baseline until they experienced an event of interest, or in those who remained event-free, until the occurrence of death, transfer away from the practice contributing their records to the CPRD, last collection of records from the practice by the CPRD, or 10 years from baseline, whichever was the earliest.

\section{Vascular risk factors}

Potential explanatory covariates were chosen by consensus between GPs and rheumatologists, to represent traditional risk factors for vascular diseases, well described in the literature, and used in previous studies of vascular disease. ${ }^{10-12}{ }^{14}$ Baseline data on presence of hypertension, hyperlipidaemia, chronic kidney disease, Body Mass Index (BMI), ever/never exposure to alcohol, smoking, statins and aspirin were identified for all participants from their EHR using relevant Read Codes. Smoking and alcohol data were entered as categorical variables, (ever/ never exposure or missing) in order to minimise the effect that missing data in the recording of these covariates would have on the size of the dataset available for analysis. Charlson Co-morbidity Index ${ }^{28}$ at baseline was also calculated using a technique described by Khan et al. ${ }^{29}$ This index is calculated based upon the presence or absence of 19 weighted comorbid conditions, including history of diabetes which was, therefore, not separately included in the multivariate analysis. Physical activity and family history of vascular disease were not included in the multivariable analysis due to high levels of missing data, and hyperuricaemia could not be included since SUA is not routinely measured in non-gout patients in the UK.

\section{Statistical analysis}

Descriptive statistics were used to describe the baseline demographics of the sample. Cox proportional hazard modelling was used to produce both unadjusted and adjusted HRs, estimating the excess risk of the various forms of vascular disease, associated with gout, ${ }^{30}$ using robust SEs to adjust for any clustering induced by matching. ${ }^{31}$ The validity of the proportional hazards assumption was tested using Schoenfeld residuals and Stata's own diagnostic test. Where this assumption was violated, suggesting the risk associated with potential explanatory covariates may vary over time, the relevant variables were reintroduced into the model as time-varying covariates. ${ }^{32}$

Baseline age, BMI, smoking and alcohol exposure and Charlson comorbidity index, along with sex and gout $\times$ sex interaction were introduced into the first model for analysis (Model 1), in order to minimise surveillance bias and represent a typical patient with gout presenting to a GP for the first time. Additionally, the second model also included baseline history of hypertension, hyperlipidaemia, chronic kidney disease and exposure to prescription statins and aspirin.

The association with vascular events was investigated by sex subset. The interaction between gout and sex was tested, with significance assessed using the Wald test, since robust SEs were used. Stratified effect sizes were calculated in order to clarify sex differences, using the STATA LINCOM command to calculate the appropriate linear combinations from the model containing the interaction. This has the added advantage of using all the data rather than fitting separate models for sex. ${ }^{33}$ Data were analysed using Stata statistical software release 12 (StataCorp: College Station, TX, 2011).

This study protocol received approval from the Independent Scientific Advisory Committee for the Medicines and Healthcare products Regulatory Agency Database Research (reference number 10_109)

\section{RESULTS}

A total of 8386 patients with gout were age, sex and practicematched to 39766 participants without gout. Mean age at diagnosis for patients with gout was 66.3 years (SD 10.8), and 69.4\% were male. Patients with gout had an increased prevalence of all risk factors of interest at baseline, with the exception of diabetes mellitus, when compared with non-gout patients (table 1).

The proportional hazards assumption for gout exposure was met for all types of vascular events. The time-varying covariates entered for each outcome are available in the online supplementary table S1.

A statistically significant interaction between gout and sex was found, and for this reason, the results are presented by sex. The details of the interaction in the gout effect between men and women are available in the online supplementary table S2.

Totally, 11266 vascular events occurred during the follow-up (table 2). Absolute risk of any vascular event per 1000 person years in men with gout was 43.63 (41.55 to 45.77) compared to 33.70 (32.86 to 34.55 ) in men without gout, corresponding to a crude HR of 1.29 (1.22 to 1.36), and in women with gout it was 51.89 (48.32 to 55.64) compared to 33.41 (32.15 to 34.71), corresponding to a HR of 1.56 (1.44 to 1.69). The most marked increase was in risk of PVD in women, where absolute risk more than doubled, from 3.05 (2.68 to 3.46) to 7.09 (5.81 to 8.55 ) events per 1000 person-years in women 
Table 1 Participant demographics at baseline

\begin{tabular}{llll}
\hline & Gout & Non-gout & p For significance \\
\hline Participants, n & 8386 & 39766 & \\
Age at diagnosis, years & $66.3( \pm 10.8)$ & $66.2( \pm 10.7)$ & 0.99 \\
Male, \% & 69.4 & 69.2 & 0.70 \\
Ever smoker (missing), \% & $28.3(23.1)$ & $26.2(31.2)$ & $<0.01$ \\
Ever drinker (missing), \% & $73.5(13.8)$ & $64.4(21.2)$ & $<0.01$ \\
BMI >25 kg/m (missing), \% & $59.7(18.3)$ & $43.6(25.0)$ & $<0.01$ \\
Hypertension, \% & 36.0 & 17.3 & $<0.01$ \\
Hyperlipidaemia, \% & 5.7 & 3.2 & $<0.01$ \\
Diabetes, \% & 4.2 & 4.4 & 0.33 \\
Chronic kidney disease, \% & 1.4 & 0.2 & $<0.01$ \\
Ever statin use, \% & 34.3 & 25.6 & $<0.01$ \\
Ever aspirin use, \% & 42.7 & 33.4 & $<0.01$ \\
\hline BMI, Body Mass Index & & &
\end{tabular}

with gout, corresponding to an unadjusted HR of 2.35 (1.87 to 2.94). There was a statistically significant increased risk of all vascular outcomes for women with gout, with the exception of MI, and for men with gout except any CVD and CVA (table 2).

In both sexes, previously identified risks remained significant, but were attenuated after adjustment for the vascular risk factors included in model 1 (table 3 ).

After adjustment for the extended range of covariates included in model 2, a clear sex difference emerged. Gout was an independent risk factor for any vascular event, any CHD and PVD, but not for any of the other vascular outcomes of interest for men. However, gout remained an independent risk factor for all types of vascular diseases in women except MI and any CVD (table 3) and, additionally, the magnitude of risk of all outcomes was found to be greater in women.

\section{DISCUSSION}

This study provides evidence that patients of both sexes with gout are at increased risk of any vascular events, any CHD and PVD even after adjustment for traditional vascular risk factors, but the magnitude of this risk is greater in women. Additionally, female gout patients are at increased risk of angina, TIA and CVA, but male patients with gout are not.

While our recent meta-analysis demonstrated an increased risk of mortality from cardiovascular causes, ${ }^{34}$ meta-analysis of risk of incident cardiovascular disease in gout has not been undertaken to date. Previous studies examining the association between gout and incident CHD have been conflicting; adjusted results from the Framingham study reported an increased incidence of CHD and angina in men but not women, but both a prospective study on male health professionals from the USA and a primary care case-control study using patients of both sexes with gout from The Netherlands, reported no increased incidence of CHD. ${ }^{10} 1415$ Studies of the association between gout and MI produce similar conflicting reports. Krishnan et $a l,{ }^{11}$ report an increased incidence of non-fatal and all MI in a male cohort nested in a controlled trial examining the efficacy of coronary risk reduction in men at high risk of vascular disease, DeVera et al, ${ }^{35}$ report an increased incidence of nonfatal and all MI in women, but not men in a retrospective cohort study in Canada, and no association between gout and $\mathrm{MI}$ in either sex reported by Abbott et al, 1988. ${ }^{10}$

The contradictory nature of current evidence may arise from significant heterogeneity of study design, and ascertainment of

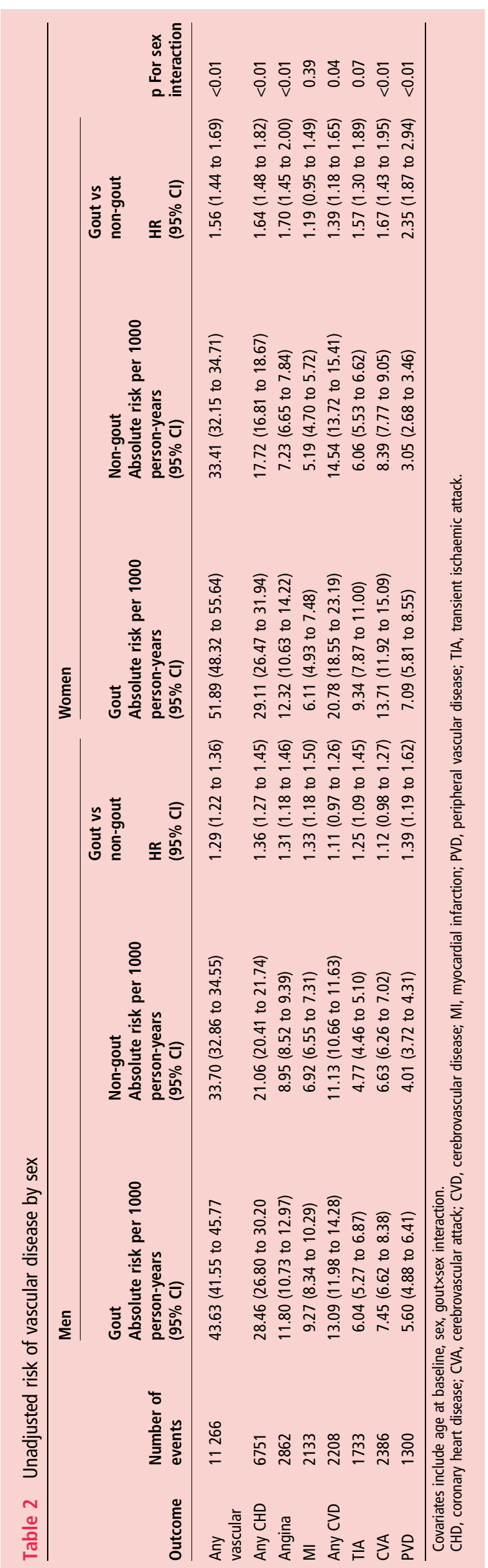


Table 3 Adjusted risk of vascular disease by sex

\begin{tabular}{|c|c|c|c|c|c|c|}
\hline \multirow[b]{2}{*}{ Outcome } & \multicolumn{3}{|l|}{ Model 1} & \multicolumn{3}{|l|}{ Model 2} \\
\hline & $\begin{array}{l}\text { HR men } \\
(95 \% \mathrm{Cl})\end{array}$ & $\begin{array}{l}\text { HR women } \\
(95 \% \mathrm{Cl})\end{array}$ & p For sex interaction & $\begin{array}{l}\text { HR men } \\
(95 \% \mathrm{Cl})\end{array}$ & $\begin{array}{l}\text { HR women } \\
(95 \% \mathrm{Cl})\end{array}$ & p For sex interaction \\
\hline Any vascular & 1.22 (1.16 to 1.29$)$ & 1.45 (1.34 to 1.57$)$ & $<0.001$ & 1.06 (1.01 to 1.12 ) & 1.25 (1.15 to 1.35$)$ & $<0.001$ \\
\hline Any CHD & 1.26 (1.18 to 1.35$)$ & 1.50 (1.35 to 1.67$)$ & 0.005 & 1.08 (1.01 to 1.15$)$ & 1.25 (1.12 to 1.39$)$ & 0.024 \\
\hline Angina & 1.20 (1.08 to 1.34$)$ & 1.55 (1.32 to 1.82$)$ & 0.010 & $1.02(0.92$ to 1.13$)$ & 1.28 (1.09 to 1.51$)$ & 0.003 \\
\hline Ml & 1.30 (1.15 to 1.46$)$ & 1.12 (0.89 to 1.40$)$ & 0.254 & 1.12 (1.00 to 1.27$)$ & 0.97 (0.77 to 1.22$)$ & 0.263 \\
\hline Any CVD & 1.11 (0.97 to 1.26 ) & 1.35 (1.14 to 1.60$)$ & 0.068 & 0.95 (0.83 to 1.09 ) & 1.17 (0.99 to 1.38 ) & 0.058 \\
\hline TIA & 1.22 (1.05 to 1.41$)$ & 1.50 (1.24 to 1.81$)$ & 0.085 & 1.02 (0.88 to 1.18 ) & 1.26 (1.05 to 1.53$)$ & 0.796 \\
\hline CVA & $1.08(0.95$ to 1.23$)$ & 1.58 (1.35 to 1.84$)$ & $<0.001$ & 0.93 (0.81 to 1.06$)$ & 1.34 (1.15 to 1.57$)$ & $<0.001$ \\
\hline PVD & 1.35 (1.16 to 1.58$)$ & 2.17 (1.73 to 2.73$)$ & $<0.001$ & 1.18 (1.01 to 1.38$)$ & 1.89 (1.50 to 2.38$)$ & 0.04 \\
\hline
\end{tabular}

Model 1 covariates: sex, gout×sex interaction, baseline age, Body Mass Index $>25 \mathrm{~kg} / \mathrm{m}^{2}$, ever/never smoking, ever/never alcohol consumption, Charlson Comorbidity Score.

Model 2 covariates include Model 1 and baseline history of hypertension, hyperlipidaemia, chronic kidney disease, ever/never statin use, ever/never aspirin use.

CHD, coronary heart disease; CVA, cerebrovascular attack; CVD, cerebrovascular disease; MI, myocardial infarction; PVD, peripheral vascular disease; TIA, transient ischaemic attack.

gout, choice of potential explanatory covariates and study populations used, all of which (with the exception of one ${ }^{35}$ ) are of smaller size than that used here, and some of which are specialised (e.g. health professionals), ${ }^{12} 14$ or solely male. ${ }^{11} 1214$

The findings of increased risk of vascular disease in females are consistent with those of two previous studies. ${ }^{16} 35$ The reasons for this increased risk remain unclear, but prolonged exposure to hyperuricaemia prior to the onset of clinical gout may play a role, since women have been observed to have an older mean age at onset of gout, lower mean SUA levels, and reduced risk of incident gout compared with men with a comparable level of hyperuricaemia. ${ }^{36-38}$ This prolonged exposure may be lengthened further if clinicians are less vigilant for gout in women, potentially delaying diagnosis and increasing crystal and inflammatory burden. Incidence of gout is higher in postmenopausal women, ${ }^{39}$ suggesting that oestrogen is an important influence, both on renal urate handling, and also on the increased incidence of abdominal obesity and associated hyperinsulinaemia after menopause which further impairs renal excretion of urate. $^{38}$ A stronger multiplicative effect between hyperuricaemia and metabolic vascular risk factors resulting in a higher propensity of women to hyperuricaemia-induced microvascular damage has also been suggested. ${ }^{40}$ However, there may simply be less aggressive management of vascular risk factors or gout itself in female primary care patients. Although there was a statistically significant difference between the proportions of male and female patients with gout prescribed allopurinol $(43 \%$ vs $39 \%, \mathrm{p}<0.01$ for difference) in our sample, a difference of this magnitude is unlikely to fully explain the sex differences seen, and further research is required in order to elucidate the nature of this relationship.

The increased incidence of PVD found in patients of both sexes with gout is also in line with the only other study to have examined this relationship. ${ }^{17}$ This association may result from common risk factors shared by gout and PVD, such as hypertension, and chronic kidney disease, ${ }^{41}$ but further research is needed to investigate this.

Our findings have several important implications for clinical practice. First, current evidence suggests that the clinical management of gout in primary care is suboptimal, ${ }^{1}{ }^{42}$ despite approximately 1 in 40 people in the UK, and over 8 million people in the USA currently affected. ${ }^{1}{ }^{2}$ Thus, even a small increase in vascular risk will give rise to a substantial number of new vascular events. Since there is evidence that cardiovascular disease in patients with gout often goes unrecognised and undertreated in primary care, with only a quarter of people consulting with acute gout screened for cardiovascular risk factors within the subsequent month, ${ }^{43}$ despite both national and international guidance recommending this, ${ }^{44-46}$ the results of this study suggest a substantial need to change practice. Second, both gout and vascular disease have historically been considered diseases of men, and so even in that minority of patients who are screened for vascular risk factors, those chosen may not be those most at risk, and since this study highlights the most serious consequences for women, perhaps more attention should be paid to prompt and reliable diagnosis of gout, followed by optimal management in female patients, including serious consideration of vascular risk reduction.

Third, screening for PVD in patients with gout is not currently recommended as part of best practice. The increased risk of incident PVD was present in both sexes, and was the strongest of those we identified. There is evidence that $44 \%$ of patients screened for PVD had PVD without evidence of $\mathrm{CHD},{ }^{47}$ suggesting that this may not have been detected by routine practice in primary care, even by those adhering to current guidance on cardiovascular screening in gout, and suggesting the need for a change in recommendations to include screening for PVD.

This study has a number of strengths compared with existing literature. The use of a large number of well-validated primary care EHR means the study is generalisable to the wider population of patients with gout. Additionally, matching patients by age, sex and GP practice reduces the risk of sociodemographic confounding. Moreover, the exclusion of patients with a prior history of vascular events reduces both surveillance bias and the additional risk conferred by a vascular history, allowing the contribution of gout itself to be more accurately investigated.

The limitations of this study include possible misclassification bias from the use of diagnostic codes to define either gout, based upon primary care diagnosis usually made on clinical grounds, or CHD/CVD outcomes of interest, where terminology may have changed over time or generalised codes may have been used. Previous studies indicate reasonable validity of gout diagnosis in primary care. ${ }^{42}$ and other studies undertaken in the CPRD have selected gout cases using a primary care diagnosis. ${ }^{1} 2348$ Similarly, a recent review of validity of diagnoses in CPRD reported a positive predictive value of diagnosis of MI coded in CPRD of over $80 \%$, with comparable reliability of coding for ischaemic heart disease to other primary care databases, ${ }^{26}$ although literature on identification of vascular diseases in EHR databases is sparse. 
While multiple adjustments on risk factors for vascular events have been performed to take into account differences between exposed and unexposed groups, we cannot rule out residual confounding effects. Similarly, while matching by registered general practice is an accepted method of accounting for sociodemographic differences, there may be considerable sociodemographic variation within a practice area that may influence this relationship. Furthermore, we were unable to adjust for hyperuricaemia (since SUA is not routinely measured in patients without gout in the UK) and family history of vascular disease and levels of physical activity in our analysis (due to poor levels of recording), which may have some bearing on this relationship. However, some degree of the effect of the variables we were unable to account for is likely to be reflected in the other risk factors included, for example, physical activity and BMI.

In conclusion, this study suggests an association between gout and subsequent CHD and PVD in both sexes, independent of traditional vascular risk factors. These risks are greatest in women, and a particularly strong association between gout and incident PVD in both sexes was found. Further work is required to establish the effect of optimum management of both vascular risk factors and gout itself on the long-term health of gout patients, clarify the nature of the relationship between gout and PVD, and the mechanism by which women are at greatest risk.

Acknowledgements We would like to thank Dr Sara Muller, Research Institute for Primary Care and Health Sciences, Keele University, for her contribution to the statistical analysis performed.

Contributors ER, SLH, JB, CH and CDM were involved in the design of the study. LEC, SLH, JB and CDM were involved in the conduct of the study. LEC and JB undertook analysis, and all authors were involved in interpretation of the data. LEC prepared the manuscript and all authors were involved in the review and approval of the final manuscript.

Funding This study is funded by the National School for Primary Care Research (grant number 88). CDM is funded by an Arthritis Research UK Clinician Scientist Award.

\section{Competing interests None.}

Provenance and peer review Not commissioned; externally peer reviewed.

Data sharing statement LEC and JB had full access to all of the data in the study and take responsibility for the integrity of the data and the accuracy of the data analysis. Additional data and codes used are available from the corresponding author on request.

Open Access This is an Open Access article distributed in accordance with the Creative Commons Attribution Non Commercial (CC BY-NC 3.0) license, which permits others to distribute, remix, adapt, build upon this work non-commercially, and license their derivative works on different terms, provided the original work is properly cited and the use is non-commercial. See: http://creativecommons.org/ licenses/by-nc/3.0/

\section{REFERENCES}

1 Kuo CF, Grainge MJ, Mallen CD, et al. Rising burden of gout in the UK but continuing suboptimal management: a nationwide population study. Ann Rheum Dis 2015;74:661-7.

2 Zhu Y, Pandya BJ, Choi HK. Prevalence of gout and hyperuricemia in the US general population: the National Health and Nutrition Examination Survey 20072008. Arthritis Rheum 2011;63:3136-41.

3 Kim SY, Guevara JP, Kim KM, et al. Hyperuricemia and coronary heart disease: a systematic review and meta-analysis. Arthritis Care Res 2010;62:170-80.

4 Kim SY, Guevara JP, Kim KM, et al. Hyperuricemia and risk of stroke: a systematic review and meta-analysis. Arthritis Rheum 2009;61:885-92.

5 Roddy E, Menon A, Hall A, et al. Polyarticular sonographic assessment of gout: a hospital-based cross-sectional study. Joint Bone Spine 2013;80:295-300.

6 Jin M, Yang F, Yang I, et al. Uric acid, hyperuricemia and vascular diseases. Front Biosci 2012;17:656-69.

7 Boogaerts MA, Hammerschmidt DE, Roelant C, et al. Mechanisms of vascular damage in gout and oxalosis: crystal induced, granulocyte mediated, endothelial injury. Thromb Haemost 1983;50:576-80.

8 Chapman PT, Yarwood H, Harrison AA, et al. Endothelial activation in monosodium urate monohydrate crystal-induced inflammation: in vitro and in vivo studies on the roles of tumor necrosis factor alpha and interleukin-1. Arthritis Rheum 1997:40:955-65.

9 Kang DH, Park SK, Lee IK, et al. Uric acid-induced C-reactive protein expression: implication on cell proliferation and nitric oxide production of human vascular cells. J Am Soc Nephrol 2005;16:3553-62.

10 Abbott RD, Brand FN, Kannel WB, et al. Gout and coronary heart disease: the Framingham Study. J Clin Epidemiol 1988;41:237-42.

11 Krishnan E, Baker JF, Furst DE, et al. Gout and the risk of acute myocardial infarction. Arthritis Rheum 2006;54:2688-96.

12 Choi HK, Curhan G. Independent impact of gout on mortality and risk of coronary heart disease. Circulation 2007;116:894-900.

13 Kuo CF, See LC, Luo SF, et al. Gout: an independent risk factor for all-cause and cardiovascular mortality. Rheumatology 2010;49:141-6.

14 Gelber AC, Klag MJ, Mead LA, et al. Gout and risk for subsequent coronary heart disease: the Meharry-Hopkins study. Arch Intern Med 1997;157:1436-40.

15 Janssens $\mathrm{HJ}$, van de Lisdonk EH, Bor $\mathrm{H}$, et al. Gout, just a nasty event or a cardiovascular signal? A study from primary care. Fam Pract 2003;20:413-16.

16 Teng GG, Ang LW, Saag KG, et al. Mortality due to coronary heart disease and kidney disease among middle-aged and elderly men and women with gout in the Singapore Chinese Health Study. Ann Rheum Dis 2012;71:924-8.

17 Baker JF, Schumacher HR, Krishnan E. Serum uric acid level and risk for peripheral arterial disease: Analysis of data from the Multiple Risk Factor Intervention Trial. Angiology 2007;58:450-7.

18 Seminog 00, Goldacre MJ. Gout as a risk factor for myocardial infarction and stroke in England: evidence from record linkage studies. Rheumatology 2013:52:2251-9.

19 Pal B, Foxall M, Dysart $T$, et al. How is gout managed in primary care? A review of current practice and proposed guidelines. Clin Rheumatol 2000;19:21-5.

20 Jordan K, Porcheret M, Kadam UT, et al. The use of general practice consultation databases in rheumatology research. Rheumatology 2006;45:126-8.

21 Smeeth L, Thomas SL, Hall AJ, et al. Risk of myocardial infarction and stroke after acute infection or vaccination. N Engl J Med 2004;351:2611-18.

22 Watson DJ, Rhodes T, Guess HA. All-cause mortality and vascular events among patients with rheumatoid arthritis, osteoarthritis, or no arthritis in the UK General Practice Research Database. J Rheumatol 2003;30:1196-202.

23 Mikuls TR, Farrar JT, Bilker WB, et al. Gout epidemiology: results from the UK General Practice Research Database, 1990-1999. Ann Rheum Dis 2005;64:267-72.

24 Tate AR, Beloff N, Al-Radwan B, et al. Exploiting the potential of large databases of electronic health records for research using rapid search algorithms and an intuitive query interface. J Am Med Inform Assoc 2014;21:292-8.

25 CPRD. http://www.cprd.com (last accessed 14 Jun 2014).

26 Khan NF, Harrison SE, Rose PW. Validity of diagnostic coding within the General Practice Research Database: a systematic review. Br J Gen Pract 2010;60:e128-36.

27 Herrett $\mathrm{E}$, Thomas SL, Schoonen WM, et al. Validation and validity of diagnoses in the General Practice Research Database: a systematic review. Br J Clin Pharmacol 2010:69:4-14.

28 Charlson $\mathrm{ME}$, Pompei $\mathrm{P}$, Ales $\mathrm{KL}$, et al. A new method of classifying prognostic comorbidity in longitudinal studies: development and validation. J Chronic Dis 1987;40:373-83.

29 Khan NF, Perera R, Harper $\mathrm{S}$, et al. Adaptation and validation of the Charlson Index for Read/OXMIS coded databases. BMC Fam Pract 2010;11:1

30 Cox D. Regression Models and Life-Tables. J R Stat Soc Ser B Metho 1972:34:187-220.

31 Rogers W. Regression standard errors in clustered samples. Stata Tech Bull (sg17) 1993;13:19-23.

32 Bellera CA, MacGrogan G, Debled M, et al. Variables with time-varying effects and the Cox-model: Some statistical concepts illustrated with a prognostic factor study in breast cancer. BMC Med Res Methodol 2010;10:20

33 http://www.stata.com/manuals13/rlincom.pdf (accessed 11 July 2013)

34 Clarson L, Chandratre P, Hider S, et al. Increased cardiovascular mortality associated with gout: a systematic review and meta-analysis. Eur J Prev Cardiol 2013 Published Online First: 26 Nov 2013. doi: 10.1177/2047487313514895

35 DeVera MA, Rahman MM, Bhole V, et al. Independent impact of gout on the risk of acute myocardial infarction among elderly women: a population-based study. Ann Rheum Dis 2010;69:1162-4.

36 Chen JH, Yeh WT, Chuang SY, et al. Gender-specific risk factors for incident gout: a prospective cohort study. Clin Rheumatol 2012;31:239-45.

37 Harrold LR, Yood RA, Mikuls TR, et al. Sex differences in gout epidemiology: evaluation and treatment. Ann Rheum Dis 2006;65:1368-72.

38 Bhole V, de Vera M, Rahman MM, et al. Epidemiology of gout in women: fifty-two-year followup of a prospective cohort. Arthritis Rheum 2010;62:1069-76.

39 Hak AE, Curhan GC, Grodstein F, et al. Menopause, postmenopausal hormone use and risk of incident gout. Ann Rheum Dis 2010;69:1305-9.

40 Cipolli JA, Ferreira-Sae MC, Martins RP, et al. Relationship between serum uric acid and internal carotid resistive index in hypertensive women: a cross-sectional study. BMC Cardiovasc Disord 2012;12:52

41 Shammas NW. Epidemiology, classification, and modifiable risk factors of peripheral arterial disease. Vasc Health Risk Manag 2007;3:229-34. 
42 Roddy E, Zhang W, Doherty M. Concordance of the management of chronic gout in a UK primary-care population with the EULAR gout recommendations. Ann Rheum Dis 2007;66:1311-15.

43 Roddy E, Mallen CD, Hider SL, et al. Prescription and comorbidity screening following consultation for acute gout in primary care. Rheumatology (Oxford) 2010:49:105-11.

44 Jordan KM, Cameron JS, Snaith M, et al.; on behalf of the British Society for Rheumatology and British Health Professionals in Rheumatology Standards, Guidelines and Audit Working Group (SGAWG). British Society for Rheumatology and British Health Professionals in Rheumatology guideline for the management of gout. Rheumatology 2007;46:1372-4.

45 Zhang W, Doherty M, Bardin T, et al. EULAR evidence based recommendations for gout. Part II: Management. Report of a task force of the EULAR Standing
Committee for International Clinical Studies Including Therapeutics (ESCISIT). Ann Rheum Dis 2006:65:1312-24.

46 Khanna D, Fitzgerald JD, Khanna PP, et al.; American College of Rheumatology. 2012 American College of Rheumatology guidelines for management of gout. Part 1:systematic nonpharmacologic and pharmacologic therapeutic approaches to hyperuricemia. Arthritis Care Res (Hoboken) 2012;64:1431-46.

47 Hirsch AT, Criqui MH, Treat-Jacobson D, et al. Peripheral arterial disease detection, awareness, and treatment in primary care. JAMA 2001;286:1317-24.

48 Mikuls TR, Farrar JT, Bilker WB, et al. Suboptimal physician adherence to quality indicators for the management of gout and asymptomatic hyperuricaemia: results from the UK General Practice Research Database (GPRD). Rheumatology (Oxford) 2005:44:1038-42. 